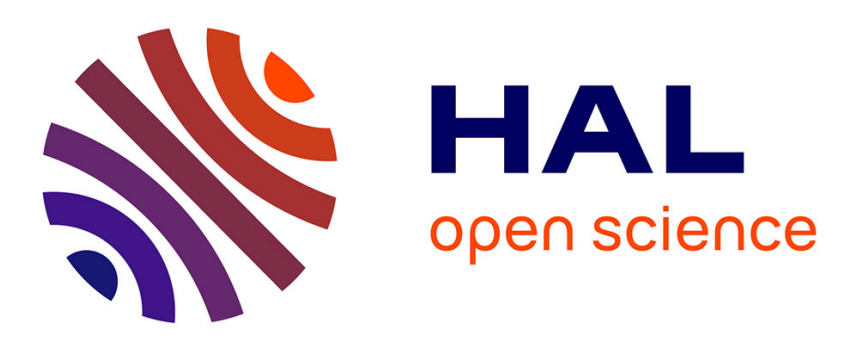

\title{
The power laws of geodesics in some random sets with dilute concentration of inclusions
}

\author{
François Willot
}

\section{To cite this version:}

François Willot. The power laws of geodesics in some random sets with dilute concentration of inclusions. Lecture Notes in Computer Science, 2015, 9082, pp.535-546. 10.1007/978-3-319-187204_45. hal-01247120v2

\section{HAL Id: hal-01247120 \\ https://hal.science/hal-01247120v2}

Submitted on 14 Feb 2019

HAL is a multi-disciplinary open access archive for the deposit and dissemination of scientific research documents, whether they are published or not. The documents may come from teaching and research institutions in France or abroad, or from public or private research centers.
L'archive ouverte pluridisciplinaire HAL, est destinée au dépôt et à la diffusion de documents scientifiques de niveau recherche, publiés ou non, émanant des établissements d'enseignement et de recherche français ou étrangers, des laboratoires publics ou privés. 


\title{
The power laws of geodesics in some random sets with dilute concentration of inclusions
}

\author{
François Willot
}

Ref.: Lecture Notes in Computer Science, 2015, Vol. 9082, pp. 535-546. This is a "post-print" version (one typo page 8 corrected, results unchanged).

\author{
Mines ParisTech, PSL Research University, \\ Center for Mathematical Morphology, \\ 35 rue $\mathrm{S}^{\mathrm{t}}$-Honoré, 77300 Fontainebleau, France, \\ francois.willot@ensmp.fr \\ WWW home page: http://cmm.ensmp.fr/ ${ }^{\sim}$ willot
}

\begin{abstract}
A method for computing upper-bounds on the length of geodesics spanning random sets in $2 \mathrm{D}$ and $3 \mathrm{D}$ is proposed, with emphasis on Boolean models containing a vanishingly small surface or volume fraction of inclusions $f \ll 1$. The distance function is zero inside the grains and equal to the Euclidean distance outside of them, and the geodesics are shortest paths connecting two points far from each other. The asymptotic behavior of the upper-bounds is derived in the limit $f \rightarrow 0$. The scalings involve powerlaws with fractional exponents $\sim f^{2 / 3}$ for Boolean sets of disks or aligned squares and $\sim f^{1 / 2}$ for the Boolean set of spheres. These results are extended to models of hyperspheres in arbitrary dimension and, in $2 \mathrm{D}$ and $3 \mathrm{D}$, to a more general problem where the distance function is non-zero in the inclusions. Finally, other fractional exponents are derived for the geodesics spanning multiscale Boolean sets, based on inhomogeneous Poisson point processes, in 2D and 3D.
\end{abstract}

Keywords: Geodesic, shortest paths, stochastic geometry, Boolean models, multiscale random sets

\section{Geodesics in random media}

Among its many applications [1], geodesics have been linked to the transport properties of nonlinear random resistor networks. In the idealized problem considered by Roux and co-workers [2,3,4], each bond in the lattice is a conductor if the voltage drop across the bond is greater than a threshold $v(\boldsymbol{x})$. The values for $v(\boldsymbol{x})$ are uniformly distributed in $[0,1]$. At the macroscopic scale, no current flows if the applied voltage is smaller than a macroscopic threshold $V$, determined by directed geodesics. More precisely:

$$
V=\min \sum_{i} v\left(\boldsymbol{x}_{i}\right)
$$


where the minimum is taken over all paths $\left(\boldsymbol{x}_{i}\right)_{i}$ spanning the lattice in the direction of the applied voltage. Similar random networks have been used to model the ductile fracture of porous materials with perfectly-plastic embedding medium [5]. In this problem, the effective plastic yield stress is determined by the length of minimal paths spanning the lattice. The length of the paths are weighted by the local plastic yield stress. Notably, several scaling laws are given in $[5,6]$ for the first-order correction to the geodesics in the 2D square network with small concentration of "porous" bonds $f \ll 1$. The latter scale as $\sim f$ for minimal paths directed parallel to the bonds and $\sim f^{1 / 2}$ along their diagonals. A scaling law $\sim f^{2 / 3}$ has been derived in 2D for the geodesics, in the continuum [7], a result consistent with numerical computations for the plastic yield stress [8].

In this article, we extend the result in [7] to other inclusion shapes, distances and dimensions. We first consider a 2D Boolean set of disks (Sec. 2), as in [7]. We derive an upper-bound for its geodesics which is sharper than the one given in [7] but coincide with the latter in the dilute limit $f \rightarrow 0$. The rest of this work is concerned by other geometries. In Sec. (3), results for the Boolean model of disks are extended to other 2D models: aligned squares (3.1), disks where the distance function is non-zero (3.2) and multiscale 2D Boolean models (3.3). Sec. (4) is devoted to 3D Boolean models of spheres (4.1), to models of spheres where the distance function is non-zero (4.2) and to multiscale 3D models (4.3). The Boolean model of hyperspheres, in arbitrary dimension, is considered in Sec. (5). We conclude in Sec. (6).

\section{Boolean set of disks}

\subsection{Distance function}

This section focuses on the geodesics, i.e. the minimal paths spanning a Boolean model [9] of disks in $\mathbb{R}^{2}$. As in [7], the distance between two points $\boldsymbol{A}$ and $\boldsymbol{B}$ is defined by:

$$
\begin{aligned}
d(\boldsymbol{A}, \boldsymbol{B})=\inf _{\boldsymbol{p} \in \mathcal{K}} \int_{0}^{1} \mathrm{~d} t \chi(\boldsymbol{p}(t))\left\|\partial_{t} \boldsymbol{p}(t)\right\|, \\
\chi(\boldsymbol{M})= \begin{cases}0 & \text { if } \boldsymbol{M} \text { lies inside a disk, } \\
1 & \text { otherwise. }\end{cases}
\end{aligned}
$$

Therefore disks are crossed at no cost whereas the embedding medium is crossed at a unit cost. In the above, $\|\cdot\|$ is the Euclidean norm, $1-\chi$ is the indicator function of the disks, and the paths are taken over the set of piecewise, continuously differentiable curves that connect $\boldsymbol{A}$ to $\boldsymbol{B}$ :

$$
\mathcal{K}=\left\{\boldsymbol{p} \in \mathcal{C}^{1}\left([0 ; 1], \mathbb{R}^{2}\right), \quad \boldsymbol{p}(0)=\boldsymbol{A}, \quad \boldsymbol{p}(1)=\boldsymbol{B}\right\} .
$$

We denote the disks surface fraction by $f(0 \leq f \leq 1)$ and their radius by $D>0$. The "dilute limit" of inclusions is the limit $f \rightarrow 0$. The disks centers follow a homogeneous Poisson point process. 
Any optimal path solution of (1) may be replaced by a union of line segments that join a set of disk centers $C^{i}(i=1, \ldots, N)$ of coordinates $\left(C_{1}^{i} ; C_{2}^{i}\right)$. We denote by $\left(\boldsymbol{A} ; \boldsymbol{C}^{1} ; \ldots ; \boldsymbol{C}^{N} ; \boldsymbol{B}\right)(N \geq 0)$ such path. We are interested in the asymptotic limit of the normalized geodesic distance:

$$
\xi=\frac{d(\boldsymbol{A}, \boldsymbol{B})}{L}, \quad L=|\boldsymbol{A} \boldsymbol{B}| \rightarrow \infty .
$$

We assume hereafter that $\boldsymbol{A}$ is the center of a disk at the origin of a Cartesian coordinate system $\left(\mathbf{e}_{1} ; \mathbf{e}_{2}\right)$ and that the line joining $\boldsymbol{A}$ and $\boldsymbol{B}$ is parallel to $\mathbf{e}_{1}$. To obtain a upper-bound on $\xi$, we follow [7] and consider a set of disk centers defined by:

$$
\begin{aligned}
& \left|C_{1}^{i+1}-C_{1}^{i}\right|=\inf \left\{\left|C_{1}-C_{1}^{i}\right| ; C\right. \text { a disk center; } \\
& \left.C_{1}>C_{1}^{i},\left|C_{2}-C_{2}^{i}\right| \leq \alpha \sqrt{D\left|C_{1}-C_{1}^{i}\right|}\right\} .
\end{aligned}
$$

where $\alpha>0$ is a constant to be optimized on. This method amounts to choosing the next disk in a domain delimited by two curves of equation $x_{2}-C_{2}^{i}=$ $\pm \alpha \sqrt{D\left|x_{1}-C_{1}^{i}\right|}$, with minimal coordinate $x_{1}$ along $\mathbf{e}_{1}$ (see Fig. 1). The shape of this domain, elongated in the $\mathbf{e}_{1}$ direction, is the result of a trade-off between following the direction $\mathbf{e}_{1}$ from $\boldsymbol{A}$ to $\boldsymbol{B}$ and maximizing the chance to find a disk at a short distance. The curves that delimit the domain, with equation $\left|C_{2}-C_{2}^{i}\right| \sim \sqrt{D\left|C_{1}-C_{1}^{i}\right|}$, follow from geometrical considerations involving three discs [7].

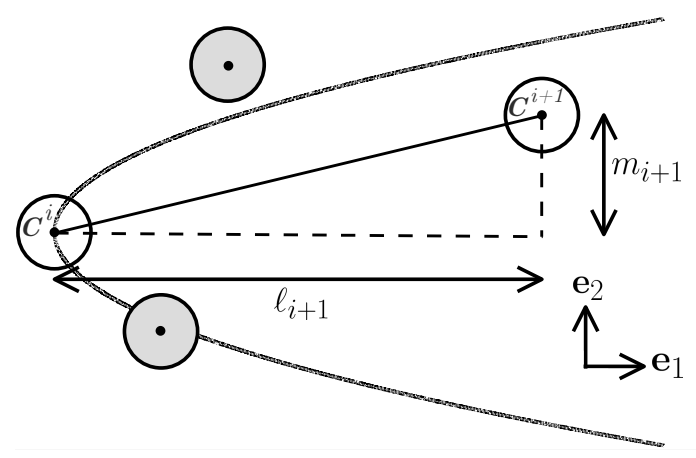

Fig. 1. Method for choosing the disk $\boldsymbol{C}^{i+1}$, knowing $\boldsymbol{C}^{i}$.

\subsection{Upper-bound on the length of geodesics}

We now compute the asymptotic length (3) of the path (4). The number $N$ is chosen so that $\boldsymbol{C}^{N+1}$ is the first disk center with coordinate along $\mathbf{e}_{1}$ larger than 
$B_{1}$, i.e. $C_{1}^{N+1}>B_{1}$ and $C_{1}^{N} \leq B_{1}$. Define $\ell_{i}=C_{1}^{i}-C_{1}^{i-1}$ and $m_{i}=C_{2}^{i}-C_{2}^{i-1}$ $(i \geq 1)$ (see Fig. 1). The path (4) provides the following upper bound on $\xi$ :

$$
\xi \leq \frac{\sum_{i=1}^{N} \max \left\{0 ; \sqrt{\ell_{i}^{2}+m_{i}^{2}}-D\right\}+Z}{\sum_{i=1}^{N} \ell_{i}}
$$

where $Z=\left|\boldsymbol{C}^{N} \boldsymbol{B}\right|$ is the Euclidean distance from $\boldsymbol{C}^{N}$ to $\boldsymbol{B}$. The $m_{i}$ are uniform random variables in the interval $\left[-\alpha \sqrt{\ell_{i} D} ; \alpha \sqrt{\ell_{i} D}\right]$. Using the Choquet capacity of a Poisson point process [10], the $\ell_{i} \in[0 ; \infty)$ follow the cumulative probability function:

$$
P\left\{\ell_{i} \leq \ell\right\}=1-(1-f)^{\frac{16 \alpha}{3 \pi}(\ell / D)^{3 / 2}} .
$$

The above yields, for the denominator in (5):

$$
\frac{1}{D N} \sum_{i=1}^{N} \ell_{i} \approx \int_{\ell \geq 0} \frac{\ell}{D} P\left\{\ell \leq \ell_{i} \leq \ell+\mathrm{d} \ell\right\}=\frac{\Gamma\left(\frac{5}{3}\right)}{4}\left[\frac{3 \pi}{-2 \alpha \log (1-f)}\right]^{2 / 3}
$$

where $\Gamma$ is the Gamma (or extended factorial) function. Its asymptotic behavior in the dilute limit $f \rightarrow 0$ reads:

$$
\frac{1}{D N} \sum_{i=1}^{N} \ell_{i}=\left(\frac{\pi}{4 \alpha \sqrt{6}}\right)^{2 / 3} \frac{\Gamma\left(\frac{2}{3}\right)}{f^{2 / 3}}+O\left(f^{1 / 3}\right) .
$$

The numerator in (5) is computed as:

$$
\begin{aligned}
\frac{1}{N} \sum_{i=1}^{N} \max \left\{0 ; \sqrt{\ell_{i}^{2}+m_{i}^{2}}-D\right\} \approx & \int_{\ell=0}^{\infty} \int_{m=0}^{\alpha \sqrt{\ell D}} \max \left\{0 ; \sqrt{\ell^{2}+m^{2}}-D\right\} \\
& \times P\left\{\ell \leq \ell_{i} \leq \ell+\mathrm{d} \ell\right\} \frac{\mathrm{d} m}{\alpha \sqrt{\ell D}} .
\end{aligned}
$$

The integration over $m$ is carried out by separating the contribution from $\ell>D$ and $\ell \leq D$. This leads to two integrals in $x=\ell / D$ with no simple analytical solution:

$$
\begin{aligned}
& \frac{1}{D N} \sum_{i=1}^{N} \max \left\{0 ; \sqrt{\ell_{i}^{2}+m_{i}^{2}}-D\right\} \approx \int_{x \geq 1} \frac{2 \mathrm{~d} x}{\pi}(1-f)^{\frac{16 \alpha x^{3} / 2}{3 \pi}} \log (1-f) \\
& \quad \times\left[4 \alpha \sqrt{x}-2 \alpha x \sqrt{\alpha^{2}+x}-x^{2} \log \left(1+\frac{2 \alpha}{x}\left(\alpha+\sqrt{\alpha^{2}+x}\right)\right)\right] \\
& \quad+\int_{x=\sqrt{1+\frac{\alpha^{4}}{4}}-\frac{\alpha^{2}}{2}}^{1} \frac{-4 \mathrm{~d} x}{\pi}(1-f)^{\frac{16 \alpha x^{3 / 2}}{3 \pi}} \log (1-f) \\
& \quad \times\left[\alpha x \sqrt{\alpha^{2}+x}+\sqrt{1-x^{2}}-2 \alpha \sqrt{x}+x^{2} \log \left(\sqrt{x} \frac{\alpha+\sqrt{\alpha^{2}+x}}{1+\sqrt{1-x^{2}}}\right)\right] .
\end{aligned}
$$


The second integral in the above equation scales as $\sim f$ when $f \rightarrow 0$. The asymptotic behavior of the first one is computed by a Taylor expansion $x \rightarrow \infty$. We find at lowest order in $f$ :

$$
\frac{1}{D N} \sum_{i=1}^{N} \max \left\{0 ; \sqrt{\ell_{i}^{2}+m_{i}^{2}}-D\right\}=\left(\frac{\pi}{4 \alpha \sqrt{6}}\right)^{2 / 3} \frac{\Gamma\left(\frac{2}{3}\right)}{f^{2 / 3}}+\frac{\alpha^{2}}{6}-1+O\left(f^{1 / 3}\right) .
$$

Note that the term in $Z$ in (5) becomes negligible when $N$ is large. Indeed, $\ell_{i} \sim f^{-2 / 3}$ from (8) and so $m_{i} \sim \sqrt{\ell_{i}} \sim f^{-1 / 3}$ and $Z$ scales as:

$$
Z=\left|\sum_{i=1}^{N} m_{i}\right| \sim \sqrt{N} f^{-1 / 3} \sim \sqrt{L}
$$

Accordingly:

$$
\frac{Z}{\sum_{i} \ell_{i}} \sim \frac{1}{\sqrt{L}} \rightarrow 0, \quad L \rightarrow \infty .
$$

Eqs. (8) and (11) then yield, for the normalized geodesic:

$$
\xi \leq 1-\frac{\alpha^{2 / 3}\left(6-\alpha^{2}\right)}{\Gamma\left(\frac{2}{3}\right)}\left(\frac{2}{3 \pi}\right)^{2 / 3} f^{2 / 3}+O\left(f^{4 / 3}\right) .
$$

The sharpest bound is obtained for $\alpha=\sqrt{3 / 2}$ :

$$
\xi \leq 1-\frac{3}{\Gamma\left(\frac{2}{3}\right)}\left(\frac{3 f}{2 \pi}\right)^{2 / 3}+O\left(f^{4 / 3}\right) \approx 1-1.3534 f^{2 / 3},
$$

a result identical to that derived in [7].

The upper-bound (5) is computed for the full range of porosity $0 \leq f \leq 1$ using (7) and (10). The two integrals in the right-hand side of (10) are solved numerically. Numerical experiments indicate that $\alpha=\sqrt{3 / 2}$ is optimal, i.e. produces the sharpest bounds, for all values of $f$. The bound is compared to numerical estimates of $\xi$ in Fig. (2b). Boolean sets with increasing disks surface fractions $f=0.06,0.11, \ldots, 0.71$ are generated on images containing $4096^{2}$ pixels. We fix the disk radius to 10 pixels and generate 10 realizations of the model for each value of $f$. We also generate 10 realizations of a model with $8192^{2}$ pixels, disks of radius 4 voxels and surface fraction 0.007. The geodesic distance is computed in each pixel of all images using Matlab's graydist function $[11,12]$. The distance between the mid-points on two opposite faces are used to estimate geodesics.

Results are represented in Fig. (2), with error bars that indicate statistical fluctuations. As expected, the upper-bound (5) is significantly higher than the exact result when the surface fraction $f$ of the disks is not small. For small values of $f$, however, the bound becomes a good estimate of the geodesics. When $f=0.007$, the upper-bound provides $\xi \leq 0.9483$ and the numerical estimate $\xi \approx 0.9470$. As expected, the upper-bound percolates at $f=1$, a value larger than the actual percolation threshold, which is about $f=f_{p}^{2 \mathrm{D}} \approx 0.68$ [13]. 


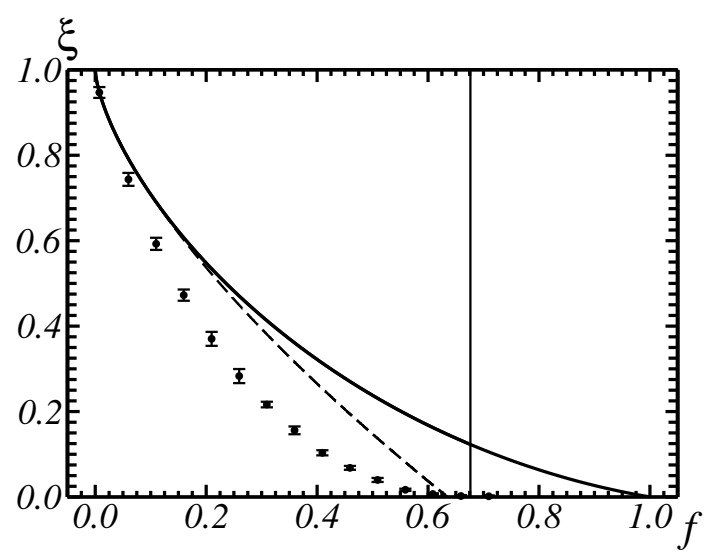

Fig. 2. Upper-bound (5) (solid line) vs. numerical estimates of $\xi$ (circles with error bars) for increasing values of the disks surface fraction $f$. Dashed line: asymptotic expansion (13). Vertical solid line: percolation threshold $f_{c} \approx 0.68$.

\section{Other 2D Boolean sets}

In this section, we extend result (13) obtained for a Boolean set of discs to some other random sets. The discs are replaced by aligned squares in Sec. (3.1). In Sec. (3.2) we let the distance function be non-zero inside the inclusions. Finally, we consider two-scales random media in Sec. (3.3).

\subsection{Boolean set of aligned squares}

In this section, we suppose that the inclusions are aligned squares of side $D$ and that points $\boldsymbol{A}$ and $\boldsymbol{B}$ are aligned with one of the direction of the squares. The path $\left(\boldsymbol{A} ; \boldsymbol{C}^{1} ; \ldots ; \boldsymbol{C}^{N} ; \boldsymbol{B}\right)$ is defined by:

$$
\begin{aligned}
& \left|C_{1}^{i+1}-C_{1}^{i}\right|=\inf \left\{\left|C_{1}-C_{1}^{i}\right| ; \quad C\right. \text { a square center; } \\
& \left.\qquad C_{1}>C_{1}^{i}+D,\left|C_{2}-C_{2}^{i}\right| \leq \alpha \sqrt{D\left|C_{1}-C_{1}^{i}\right|}\right\} .
\end{aligned}
$$

Note that compared to Eq. (4), the $\boldsymbol{C}^{i}$ are now square centers and we have added the condition $C_{1}>C_{1}^{i}+D$. Like in Sec. (2.2), we set $\ell_{i}=C_{1}^{i}-C_{1}^{i-1}$, $m_{i}=C_{2}^{i}-C_{2}^{i-1}$ with $\ell_{i} \geq D,\left|m_{i}\right| \leq \alpha \sqrt{\ell_{i} D}$. Again, $\alpha$ is a constant to be optimized on and we denote $f \ll 1$ the surface fraction of the squares. The distance function $d_{\mathrm{sq}}$ is defined as in (1) with $\chi$ replaced by the indicator function of the Boolean set of squares. Note that:

$$
d_{\mathrm{sq}}\left(\boldsymbol{C}^{i-1}, \boldsymbol{C}^{i}\right) \leq \sqrt{\left(\ell_{i}-D\right)^{2}+\left(m_{i}-D\right)^{2}},
$$

which yields for the normalized geodesic distance:

$$
\xi_{\mathrm{sq}}=\frac{d_{\mathrm{sq}}(\boldsymbol{A}, \boldsymbol{B})}{L} \leq \frac{Z+\sum_{i=1}^{N} \sqrt{\left(\ell_{i}-D\right)^{2}+\left(m_{i}-D\right)^{2}}}{\sum_{i=1}^{N} \ell_{i}},
$$


with $Z=\left|\boldsymbol{C}^{N} \boldsymbol{B}\right|, L=|\boldsymbol{A} \boldsymbol{B}|$. For squares, Eq. (6) now takes the form:

$$
P\left\{\ell_{i} \leq \ell\right\}=1-(1-f)^{\frac{4 \alpha}{3}\left[(\ell / D)^{3 / 2}-1\right]}
$$

and the average of the $\ell_{i}$ reads:

$$
\begin{aligned}
\frac{1}{N} \sum_{i=1}^{N} \frac{\ell_{i}}{D} & \approx 1+\frac{2}{3}(1-f)^{-\frac{4 \alpha}{3}} E_{1 / 3}\left(\frac{-4 \alpha \log (1-f)}{3}\right) \\
& =\frac{\Gamma\left(\frac{2}{3}\right)}{(\sqrt{6} \alpha f)^{2 / 3}}+O\left(f^{1 / 3}\right), \quad f \rightarrow 0 .
\end{aligned}
$$

The mean of the term $\sqrt{\left(\ell_{i}-D\right)^{2}+\left(m_{i}-D\right)^{2}}$ occurring in (15) is expressed as a double integral like in Sec. (2.2). We develop it for $\ell_{i} \gg D$ and integrate over $\ell_{i}$ and $m_{i}$ :

$$
\frac{1}{D N} \sum_{i=1}^{N} \sqrt{\left(\ell_{i}-D\right)^{2}+\left(m_{i}-D\right)^{2}}=\frac{\Gamma\left(\frac{2}{3}\right)}{(\sqrt{6} \alpha f)^{2 / 3}}+\left(\frac{\alpha^{2}}{6}-1\right)+O\left(f^{1 / 3}\right) .
$$

We neglect $Z$ and choose $\alpha=\sqrt{3 / 2}$ :

$$
\xi_{\mathrm{sq}} \leq 1-\frac{3^{5 / 3}}{4 \Gamma\left(\frac{2}{3}\right)} f^{2 / 3}+o\left(f^{2 / 3}\right) \approx 1-1.1521 f^{2 / 3}
$$

This correction is smaller than that derived for the disks model. We emphasize that this model is anisotropic and that the geodesics are directed parallel to the squares's side. The correction is larger for geodesics oriented along the diagonal.

\subsection{Boolean set of disks with non-zero distance function inside the disks}

In this section, we consider a Boolean model of disks with the following modified distance function:

$$
\begin{aligned}
d_{p}(\boldsymbol{A}, \boldsymbol{B})= & \inf _{\boldsymbol{p} \in \mathcal{K}} \int_{0}^{1} \mathrm{~d} t \chi_{p}(\boldsymbol{p}(t))\left\|\partial_{t} \boldsymbol{p}(t)\right\| \\
& \chi_{p}(\boldsymbol{M})=\left\{\begin{array}{lr}
\mathrm{p} & \text { if } \boldsymbol{M} \text { lies inside a disk } \\
1 & \text { otherwise }
\end{array}\right.
\end{aligned}
$$

where $0 \leq p<1$ is the cost associated to the distance in the disks. The distance function $d$ in (1) is recovered when $p=0$. We consider a similar path $\left(\boldsymbol{A} ; \boldsymbol{C}^{1} ; \ldots ; \boldsymbol{C}^{N} ; \boldsymbol{B}\right)$ as in Sec. $(2.2)$, defined by:

$$
\begin{aligned}
& \left|C_{1}^{i+1}-C_{1}^{i}\right|=\inf \left\{\left|C_{1}-C_{1}^{i}\right| ; \quad C\right. \text { a disk center; } \\
& \left.\qquad C_{1}>C_{1}^{i}+D,\left|C_{2}-C_{2}^{i}\right| \leq \alpha \sqrt{D\left|C_{1}-C_{1}^{i}\right|}\right\} .
\end{aligned}
$$


with the extra condition $C_{1}>C_{1}^{i}+D$. It provides a bound on $d_{p}(\boldsymbol{A}, \boldsymbol{B})$ :

$$
\xi_{p}=\frac{d_{p}(\boldsymbol{A}, \boldsymbol{B})}{L} \leq \frac{\sum_{i=1}^{N}\left[\sqrt{\ell_{i}^{2}+m_{i}^{2}}-(1-p) D\right]}{\sum_{i=1}^{N} \ell_{i}},
$$

with $Z=\left|\boldsymbol{C}^{N} \boldsymbol{B}\right|, \ell_{i}=C_{1}^{i}-C_{1}^{i-1}, m_{i}=C_{2}^{i}-C_{2}^{i-1}, \ell_{i} \geq D,\left|m_{i}\right| \leq \alpha \sqrt{\ell_{i} D}$ and $\alpha>0$. Note that the path $\left(\boldsymbol{A} ; \boldsymbol{C}^{1} ; \ldots ; \boldsymbol{C}^{N} ; \boldsymbol{B}\right)$ used to derive bound (22) consists in a set of segments joining the disks centers. Most geodesics will not pass through disk centers when $p>0$. Nevertheless, in the dilute regime considered here we expect $\left|m_{i}\right| \ll \ell_{i}$ so that bound (22) should be a very good estimate of the length of the path $\left(\boldsymbol{A} ; \boldsymbol{C}^{1} ; \ldots ; \boldsymbol{C}^{N} ; \boldsymbol{B}\right)$. The asymptotic expansions for the means of the $\ell_{i}$ and of the quantity $\sqrt{\ell_{i}^{2}+m_{i}^{2}}-D$ are the same as in (11) and (8). This yields:

$$
\begin{aligned}
\xi_{\mathrm{p}} & \leq 1-\left(\frac{2 \alpha}{3 \pi}\right)^{2 / 3} \frac{6(1-p)-\alpha^{2}}{\Gamma\left(\frac{2}{3}\right)} f^{2 / 3}+o\left(f^{2 / 3}\right), \\
& =1-\frac{3(1-p)^{4 / 3}}{\Gamma\left(\frac{2}{3}\right)}\left(\frac{3}{2 \pi}\right)^{2 / 3} f^{2 / 3}+o\left(f^{2 / 3}\right) \approx 1.3534(1-p)^{4 / 3} f^{2 / 3},
\end{aligned}
$$

with $\alpha=\sqrt{3(1-p) / 2}$. The upper-bound above is sharper than the trivial bound $\xi_{\mathrm{p}} \leq 1-(1-p) f$ in the domain $f \ll 1-p$.

\subsection{Multiscale Boolean set of disks}

Consider first two Boolean sets of disks with constant diameter, denoted $\mathcal{M}_{1}$ and $\mathcal{M}_{2}$. The disks of set $\mathcal{M}_{1}$ have constant diameter $D_{1}$ and that of set $\mathcal{M}_{2}$ have diameter $D_{2} \ll D_{1}$. The centers of the disks in the Boolean sets $\mathcal{M}_{1}$ and $\mathcal{M}_{2}$ follow a homogeneous Poisson point process. We denote by $f_{1}$ and $f_{2}$ the surface fractions of sets $\mathcal{M}_{1}$ and $\mathcal{M}_{2}$ respectively and assume $f_{1} \ll 1, f_{2} \ll 1$. Hereafter we consider the intersection of the two sets $\mathcal{M}=\mathcal{M}_{1} \cap \mathcal{M}_{2}$ which is a two-scales random set with surface fraction $f=f_{1} f_{2}$. The model is assumed "symmetric" so that $f_{1}=f_{2}=\sqrt{f}$ and the distance function defined as in (1).

Accordingly to (13), the distance $d\left(\boldsymbol{A}^{\prime}, \boldsymbol{B}^{\prime}\right)$ between two points $\boldsymbol{A}^{\prime}$ and $\boldsymbol{B}^{\prime}$ that lie in a disk contained in $\mathcal{M}_{1}$ admits the following upper bound:

$$
\frac{d\left(\boldsymbol{A}^{\prime}, \boldsymbol{B}^{\prime}\right)}{L^{\prime}} \leq 1-\frac{3}{\Gamma\left(\frac{2}{3}\right)}\left(\frac{3}{2 \pi}\right)^{2 / 3} f_{2}^{2 / 3}+o\left(f_{2}^{2 / 3}\right),
$$

when $L^{\prime}=\left|\boldsymbol{A}^{\prime} \boldsymbol{B}^{\prime}\right| \gg D_{2}$. In the limit $D_{2} \ll D_{1}$ the distance function in $\mathcal{M}$ is well approximated by that considered in Sec. (3.2) with $p=1-\frac{3}{\Gamma\left(\frac{2}{3}\right)}\left(\frac{3 f_{2}}{2 \pi}\right)^{2 / 3}$. Eq. (24) then provides the following bound, for two points $\boldsymbol{A}$ and $\boldsymbol{B}$ sufficiently far away from each other:

$$
\xi_{\mathrm{ms}}=\frac{d(\boldsymbol{A}, \boldsymbol{B})}{L} \leq 1-\frac{3^{35 / 9}}{(2 \pi)^{14 / 9} \Gamma\left(\frac{2}{3}\right)^{7 / 3}} f^{7 / 9}+o\left(f^{7 / 9}\right) \approx 1-2.0261 f^{7 / 9} .
$$


This correction is smaller than that derived in the one-scale model (13) and indicates that clustering tend to "constraint" the shortest paths and increase their lengths. Similarly, the 2D periodic model, which has a very homogeneous spatial distribution of voids, has an exponent $1 / 2$ and its geodesics are shorter than in the one-scale Boolean set of disks.

\section{3D Boolean sets}

\subsection{Boolean set of spheres}

In this section, we study a Boolean set of spheres of volume fraction $f$. The distance function $d^{3 \mathrm{D}}$ is defined in $3 \mathrm{D}$ as in (1):

$$
d^{3 \mathrm{D}}(\boldsymbol{A}, \boldsymbol{B})=\inf _{\boldsymbol{p} \in \mathcal{K}} \int_{0}^{1} \mathrm{~d} t \chi^{3 \mathrm{D}}(\boldsymbol{p}(t))\left\|\partial_{t} \boldsymbol{p}(t)\right\|
$$

with $1-\chi^{3 \mathrm{D}}$ is the indicator of the Boolean set of spheres and $\mathcal{K}$ is given by (2). We define the path $\left(\boldsymbol{C}^{0}=\boldsymbol{A} ; \boldsymbol{C}^{1} ; \ldots ; \boldsymbol{C}^{N} ; \boldsymbol{C}^{N+1}=\boldsymbol{B}\right)$ by:

$$
\begin{aligned}
& \left|C_{1}^{i+1}-C_{1}^{i}\right|=\inf \left\{\left|C_{1}-C_{1}^{i}\right| ; \boldsymbol{C}\right. \text { a sphere center; } \\
& \left.\qquad C_{1}>C_{1}^{i}+D,\left|\boldsymbol{C}-\boldsymbol{C}^{\prime}\right| \leq \alpha \sqrt{D\left|C_{1}-C_{1}^{i}\right|}\right\},
\end{aligned}
$$

where $\boldsymbol{C}^{\prime}$ is the orthogonal projection of $\boldsymbol{C}$ onto the line $\left(\boldsymbol{C}^{i} ; \boldsymbol{C}^{i}+\mathbf{e}_{1}\right)$ and $\alpha$ is a constant to be optimized on. As in Sec. (2), the axis $\mathbf{e}_{1}$ is aligned with the line passing by $\boldsymbol{A}$ and $\boldsymbol{B}$. We set $\ell_{i}=C_{1}^{i}-C_{1}^{i-1}$ and $m_{i}=\left|\boldsymbol{C}^{i} \boldsymbol{C}^{i^{\prime}}\right|$ so that $\left|\boldsymbol{C}^{i-1} \boldsymbol{C}^{i}\right|^{2}=\ell_{i}^{2}+m_{i}^{2}$. The path (27) leads to the bound:

$$
\xi^{3 \mathrm{D}}=\frac{d^{3 \mathrm{D}}(\boldsymbol{A}, \boldsymbol{B})}{L} \leq \frac{\sum_{i=1}^{N}\left(\sqrt{\ell_{i}^{2}+m_{i}^{2}}-D\right)+Z}{\sum_{i=1}^{N} \ell_{i}}
$$

with $Z=\left|\boldsymbol{C}^{N} \boldsymbol{B}\right|$. Using the Choquet capacity [10], the variables $\ell_{i} \in[D ; \infty)$ follow the cumulative probability function according t:

$$
P\left\{\ell_{i} \leq \ell\right\}=1-(1-f)^{3 \alpha^{2}\left[(\ell / D)^{2}-1\right]} .
$$

and so, for the mean of the $\ell_{i}$ :

$$
\begin{aligned}
\frac{1}{D N} \sum_{i=1}^{N} \ell_{i} & \approx 1+\frac{(1-f)^{-3 \alpha^{2}}}{2 \alpha} \sqrt{\frac{\pi}{-3 \log (1-f)}} \operatorname{erfc}(\alpha \sqrt{-3 \log (1-f)}) \\
& =\frac{1}{2 \alpha} \sqrt{\frac{\pi}{3 f}}+O(\sqrt{f}), \quad f \rightarrow 0,
\end{aligned}
$$


where $\operatorname{erfc}(z)=2 / \sqrt{\pi} \int_{z}^{\infty} \mathrm{d} t \mathbf{e}^{-t^{2}}$ is the complementary error function. The mean of the quantity $\sqrt{\ell_{i}^{2}+m_{i}^{2}}$ reads:

$$
\begin{aligned}
\frac{1}{N} \sum_{i=1}^{N} \frac{\sqrt{\ell_{i}^{2}+m_{i}^{2}}}{D} & \approx \int_{\ell=D}^{\infty} \int_{m=0}^{\alpha \sqrt{\ell D}} \sqrt{\ell^{2}+m^{2}} P\left\{\ell \leq \ell_{i} \leq \ell+\mathrm{d} \ell\right\} \frac{2 m \mathrm{~d} m}{\alpha^{2} \ell D^{2}} \\
& =-\int_{x=1}^{\infty} \mathrm{d} x 4 x^{3 / 2} \log (1-f)(1-f)^{3 \alpha^{2}\left(x^{2}-1\right)}\left[\left(x+\alpha^{2}\right)^{3 / 2}-x^{3 / 2}\right] \\
& =\frac{1}{2 \alpha} \sqrt{\frac{\pi}{3 f}}+\frac{\alpha^{2}}{4}+O(\sqrt{f}),
\end{aligned}
$$

where the expression behind the integral has been expanded for $x \rightarrow \infty$. With $\alpha=2 / \sqrt{3}$ and $Z \ll 1$ :

$$
\xi^{3 \mathrm{D}} \leq 1-\frac{8}{3 \sqrt{\pi}} \sqrt{f}+o(\sqrt{f}) \approx 1-1.5045 \sqrt{f}, \quad f \rightarrow 0
$$

The number of "possible choices" for picking $\boldsymbol{C}^{i+1}$ knowing $\boldsymbol{C}^{i}$ is greater in 3D than in $2 \mathrm{D}$ and results in a lower exponent in $3 \mathrm{D}$.

\subsection{Non-zero distance function in spheres}

The reasoning above extends to a distance function $d_{\mathrm{p}}^{3 \mathrm{D}}$ which is non-zero in the spheres. Define the distance $d_{\mathrm{p}}^{3 \mathrm{D}}$ as in $(26)$ with $\chi^{3 \mathrm{D}}$ replaced by:

$$
\chi_{\mathrm{p}}^{3 \mathrm{D}}(\boldsymbol{M})=\left\{\begin{array}{l}
\mathrm{p} \text { if } \boldsymbol{M} \text { lies inside a sphere } \\
1
\end{array}\right.
$$

where $0 \leq p<1$ is a parameter. The following upper-bound on the normalized shortest paths is derived:

$$
\xi_{\mathrm{p}}^{3 \mathrm{D}}=\frac{d_{\mathrm{p}}^{3 \mathrm{D}}(\boldsymbol{A}, \boldsymbol{B})}{|\boldsymbol{A} \boldsymbol{B}|} \leq 1-\frac{8(1-p)^{3 / 2}}{3 \sqrt{\pi}} \sqrt{f}+o(\sqrt{f}), \quad f \rightarrow 0 .
$$

Again, this bound is non-trivial when $f \ll 1-p$.

\subsection{Multiscale Boolean set of spheres}

The two-scales "symmetric" Boolean set of spheres is constructed similarly as in Sec. (3.3). The latter is the intersection of two Boolean models of spheres with scale separation. The indicator function $\chi_{\mathrm{ms}}^{3 \mathrm{D}}$ of the two-scales Boolean model is used to define the distance $d_{\mathrm{ms}}^{3 \mathrm{D}}$ as in (26). The normalized shortest path is expanded in the dilute limit as:

$$
\xi_{\mathrm{ms}}^{3 \mathrm{D}}=\frac{d_{\mathrm{ms}}^{3 \mathrm{D}}(\boldsymbol{A}, \boldsymbol{B})}{|\boldsymbol{A} \boldsymbol{B}|} \leq 1-\left(\frac{8}{3 \sqrt{\pi}}\right)^{5 / 2} f^{5 / 8}+o\left(f^{5 / 8}\right) \approx 1-2.7764 f^{5 / 8}, \quad f \rightarrow 0 .
$$


Our conclusions are the same as that given in 2D. At fixed volume fraction of pores, geodesics are higher in the two-scales symmetric model than in the one-scale Boolean model. The shortest geodesics are found for the 3D periodic model, in which the spatial distribution of voids is very homogeneous and the voids well-separated.

\section{Boolean model of hyperspheres}

In this section we consider a Boolean model of hyperspheres in dimension $d \geq 2$. The geodesic distance $d^{\mathrm{dD}}$ and path $\left(\boldsymbol{A} ; \boldsymbol{C}^{1} ; \ldots ; \boldsymbol{C}^{N} ; \boldsymbol{B}\right)$ are defined as in $(26)$ and (27) with spheres replaced by hyperspheres. A bound for the geodesic is given by the path from $\boldsymbol{A}$ to $\boldsymbol{B}$. With $\ell_{i}=C_{1}^{i}-C_{1}^{i-1}, m_{i}=\left|\boldsymbol{C}^{i} \boldsymbol{C}^{i^{\prime}}\right|, Z=\left|\boldsymbol{C}^{N} \boldsymbol{B}\right|$ and $\boldsymbol{C}^{\prime}$ the orthogonal projection of $\boldsymbol{C}$ onto the line $\left(\boldsymbol{C}^{i} ; \boldsymbol{C}^{i}+\mathbf{e}_{1}\right)$, we have:

$\xi^{\mathrm{dD}}=\frac{d^{\mathrm{dD}}(\boldsymbol{A}, \boldsymbol{B})}{L} \leq \frac{\sum_{i=1}^{N}\left(\sqrt{\ell_{i}^{2}+m_{i}^{2}}-D\right)+Z}{\sum_{i=1}^{N} \ell_{i}} \approx 1-\frac{-Z+\sum_{i=1}^{N}\left(D-\frac{m_{i}^{2}}{2 \ell_{i}}\right)}{\sum_{i=1}^{N} \ell_{i}}$.

Denote by $\pi_{d}(D)$ the volume of the hyperdimensional ball of diameter $D$ and $V_{\ell}$ that of the domain:

$$
\left\{C ; \quad D \leq C_{1} \leq \ell, \quad 0 \leq\left|C^{\prime}\right| \leq \alpha \sqrt{\ell D}\right\} .
$$

The probability law for the $\ell_{i} \in\left[D, \infty\right.$ (depends on $V_{\ell}$ by:

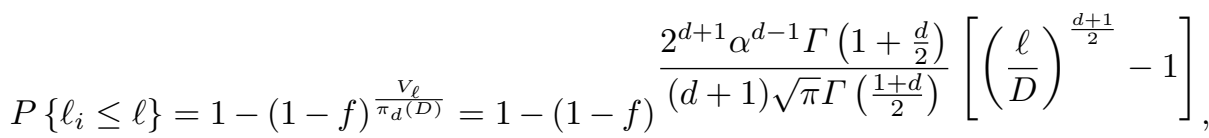

which provides the sum:

$$
\frac{1}{D N} \sum_{i=1}^{N} \frac{m_{i}^{2}}{\ell_{i}} \approx \int_{\substack{\ell \geq D, m \leq \alpha \sqrt{\ell D}}} P\left\{\ell \leq \ell_{i} \leq \ell+\mathrm{d} \ell\right\} \frac{(d-1) m^{d} \mathrm{~d} m}{\alpha^{d-1}(D \ell)^{\frac{d+1}{2}}}=\alpha^{2} \frac{d-1}{d+1} .
$$

The mean of the $\ell_{i}$, approximated by $\int_{\ell>D} \ell P\left\{\ell \leq \ell_{i} \leq \ell+\mathrm{d} \ell\right\}$, is determined using the symbolic solver Mathematica [14]. The expression involves the function $\Gamma$ as well as incomplete $\Gamma$ functions (not shown). Carrying out a Taylor expansion of the latter and optimizing on $\alpha$ yield $\alpha=\sqrt{(1+d) / d}$. Finally:

$$
\xi^{\mathrm{dD}} \leq 1-\frac{(d+1)^{\frac{1+3 d}{1+d}}}{\Gamma\left(\frac{2}{1+d}\right)}\left[\frac{\Gamma\left(1+\frac{d}{2}\right)}{2 \sqrt{\pi} d^{d} \Gamma\left(\frac{3+d}{2}\right)}\right]^{\frac{2}{1+d}} f^{\frac{2}{1+d}}+o\left(f^{\frac{2}{1+d}}\right), \quad f \rightarrow 0 .
$$

The above generalizes (13) and (32). Taking successively the limits $f \rightarrow 0$ and $d \rightarrow \infty$ :

$$
\xi^{\mathrm{dD}} \leq 1-2\left(1-\frac{\log (2 d \pi)-2(1+\gamma)}{d}\right) f^{\frac{2}{1+d}} \sim 1-\left(\frac{f}{\sqrt{f_{p}}}\right)^{2 / d}
$$


where $\gamma \approx 0.5772$ is Euler's constant and $f_{p} \sim 2^{-d}$ is the asymptotic percolation threshold in dimension $d \gg 1[15]$.

\section{Conclusion}

Powerlaws with fractional exponents $2 / 3$ and $1 / 2$ have been derived for the lowest-order corrections to the lengths of geodesics in 2D and 3D Boolean models of discs and spheres, respectively. The method is general and provides an upperbound with lowest-order correction $\sim f^{\frac{2}{d-1}}$ in dimension $d \geq 2$.

The bounds obtained for multiscale models, which scale as $\sim f^{7 / 9}$ in $2 \mathrm{D}$ and $\sim f^{5 / 8}$ indicate lower variations of the geodesics near the point $f=0$. These results underline that the the singularities for the geodesics are small for highly-heterogeneous dispersion of particles, and high when the dispersion is homogeneous.

Acknowledgement This study was made with the support of A.N.R. (Agence Nationale de la Re- cherche) under grant 20284 (LIMA project).

\section{References}

1. Li, F., Klette, R., Euclidean shortest paths - exact or approximate algorithms, Springer, London (2011).

2. Roux, S., Herrmann, H. J.: Disordered-induced nonlinear conductivity. Europhys. Lett. 4(11), 1227-1231 (1987).

3. Roux, S., Hansen, A., Guyon, É.: Criticality in non-linear transport properties of heterogeneous materials. J. Physique 48(12), 2125-2130 (1987).

4. Roux, S., Herrmann, H., Hansen, A., Guyon, É.: Relation entre différents types de comportements non linéaires de réseaux désordonnés. C. R. Acad. Sci. série II 305(11), 943-948 (1987).

5. Roux, S., François, D.: A simple model for ductile fracture of porous materials. Scripta Metall. Mat. 25(5), 1087-1092 (1991).

6. Derrida, B., Vannimenus, J.: Interface energy in random systems. Phys. Rev. B 27(7), 4401 (1983).

7. Willot, F.: The power law of geodesics in $2 \mathrm{D}$ random media with dilute concentration of disks. Submitted to Phys. Rev. E.

8. Willot, F.: Contribution à l'étude théorique de la localisation plastique dans les poreux. Diss., École Polytechnique (2007).

9. Matheron, G., Random sets and integral geometry, Wiley, New-York (1975).

10. Matheron, G.: Random sets theory and its applications to stereology. J. Microscopy 95(1), 15-23 (1972).

11. MATLAB and Statistics Toolbox Release 2012b, The MathWorks, Inc., Natick, Massachusetts, United States.

12. Soille, P.: Generalized geodesy via geodesic time. Pat. Rec. Let. 15(12), 1235-1240 (1994).

13. Quintanilla, J., Torquato, S., Ziff, R. M: Efficient measurement of the percolation threshold for fully penetrable discs. J. Phys. A: Math. Gen. 33(42), L399 (2000).

14. Wolfram Research, Inc.: Mathematica, Version 10.0. Champaign, IL (2014).

15. Torquato, S.: Effect of dimensionality on the continuum percolation of overlapping hyperspheres and hypercubes. J. of Chem. Phys. 136(5), 054106 (2012). 